\title{
Unitary Equivalence of Temperature Dynamics for Ideal and Locally Perturbed Fermi-Gas
}

\author{
D. D. Botvich and V. A. Malyshev \\ Department of Mathematics, Moscow State University, Moscow V-234, USSR
}

\begin{abstract}
We consider the local perturbation

$$
V=\varepsilon \sum_{x, y \in \mathbb{Z}^{v}} V(x, y) \chi_{\Omega}(x) \chi_{\Omega}(y) a^{*}(x) a^{*}(y) a(y) a(x)
$$

of the ideal Fermi-gas on the lattice $\mathbb{Z}^{v}$, where $\Omega$ is a finite subset of $\mathbb{Z}^{v}$ and $\chi_{\Omega}$ is its indicator. The invertibility of Möller morphisms for small $\varepsilon$ is proven. It follows that in the cyclic GNS representation with respect to KMS states the dynamics of ideal and locally perturbed Fermi-gas are unitary equivalent.
\end{abstract}

\section{Introduction}

Two kinds of equilibrium states are usually considered in mathematical physics: ground (zero temperature) and KMS (nonzero temperature) states. There are many results concerning spectral decompositions of Hamiltonians (in the GNSrepresentation) for the ground state representations which support the so-called quasi-particle picture: any system is a collection of noninteracting quasiparticles (we note that asymptotic completeness is not proven even for the ground state representations).

In Appendix B we explicitly calculate spectral decomposition of $H_{0}$ showing the quasiparticle picture. We could not find this representation in the literature.

For the KMS-states the only results in this direction are due to Robinson, Evans etc. [1-3], who proved the existence of Möller morphisms for local perturbations of quasi-free systems. In this paper we prove the invertibility of these morphisms. An extension of our method and other results will appear in subsequent publications.

\section{Formulation of the Main Result}

Let $K=l_{2}\left(\mathbb{Z}^{\nu}\right)$ be a complex Hilbert space and $\mathfrak{U}=\mathfrak{U}(K)$ be the CAR-algebra over $K$. It is well known that its generators $a(f), a^{*}(f), f \in K$ satisfy the following 
anticommutation relations

$$
\begin{gathered}
a^{*}(g) a(f)+a(f) a^{*}(g)=(f, g) I, \\
a(f) a(g)+a(g) a(f)=0,
\end{gathered}
$$

where $(f, g)$ is the scalar product in $K$ antilinear in $f$ and linear in $g$ (see [3]).

We consider the free evolution of the ideal Fermi-gas. It is a strongly continuous one-parametric group $\tau_{t}$ of *-automorphisms of $\mathfrak{U}$ generated by

$$
\tau_{t}(a(f))=a\left(e^{i t H} f\right), \quad \tau_{t}\left(a^{*}(f)\right)=a^{*}\left(e^{i t H} f\right)
$$

where $H=-\Delta+\mu 1_{K}$ acts in $K$ (lattice Laplacian plus a constant, see [3]).

Let us consider also the perturbed dynamics (local dynamical perturbation [1])

$$
\begin{aligned}
\tau_{t}^{V}(A)= & \tau_{t}(A)+\sum_{n=1}^{\infty} i^{n} \int_{0 \leqq s_{1} \leqq \ldots \leqq s_{n} \leqq t} d s_{1} \ldots d s_{n} \\
& \cdot\left[\tau_{s_{1}}(V),\left[\tau_{s_{2}}(V),\left[\ldots\left[\tau_{s_{n}}(V), \tau_{t}(A)\right] \ldots\right]\right]\right]
\end{aligned}
$$

for $t \geqq 0$ and in similarly for $t \leqq 0$ with the integration domain : $t \leqq s_{n} \leqq \ldots \leqq s_{1} \leqq 0$.

Here $V \in \mathfrak{U}$ and further on we consider only the case of

$$
V=\varepsilon \sum_{x, y \in \mathbb{Z}^{v}} V_{\Omega}(x, y) a_{x}^{*} a_{y}^{*} a_{y} a_{x}
$$

where $V_{\Omega}(x, y)$ is a real symmetric function with finite support $\Omega \times \Omega, \Omega \subset \mathbb{Z}^{v}$

$$
a_{x}=a\left(\delta_{x}\right), \quad \delta_{x}(y)= \begin{cases}1, & y=x \\ 0, & y \neq x .\end{cases}
$$

Theorem 1. Möller morphisms

$$
\gamma_{ \pm}(A)=\operatorname{sim}_{t \rightarrow \pm \infty} \tau_{-t}^{V}\left(\tau_{t}(A)\right)
$$

exist for any $A \in \mathfrak{A}$, any $v \geqq 1, \varepsilon \in \mathbb{R}, V, \Omega$.

The main result of the paper is the following:

Theorem 2. For $v \geqq 3$ and any $V_{\Omega}$, one can find $\varepsilon_{0}=\varepsilon_{0}\left(V_{\Omega}, v\right)$ such that for any $|\varepsilon|<\varepsilon_{0}$ there exist

$$
\bar{\gamma}_{ \pm}(A)=\operatorname{sim}_{t \rightarrow \pm \infty} \tau_{-t}\left(\tau_{t}^{V}(A)\right)
$$

for any $A \in \mathfrak{A}$.

Let $\omega_{0}$ be the unique $\left(\tau_{t, \beta}\right)$-KMS-state $(0<\beta<\infty)$ for the ideal Fermi-gas (see [3]). Let $\omega_{V}$ be the unique (see [3, p. 161]) $\left(\tau_{t, \beta}^{V}\right)$-KMS-state defined by

$$
\omega_{V}(A)=\frac{\omega_{0}\left(\Gamma^{*} A \Gamma\right)}{\omega_{0}\left(\Gamma^{*} \Gamma\right)},
$$


where the co-cycle $\Gamma\left(\right.$ formally $\left.\Gamma=e^{-\frac{\beta}{2}(d \Gamma(H)+V)} e^{\frac{\beta}{2} d \Gamma(H)}\right)$ is given by the convergent series [3]

$$
\Gamma=I+\sum_{n=1}^{\infty}(-1)^{n} \int_{0}^{\beta / 2} d s_{1} \int_{0}^{s_{1}} d s_{2} \ldots \int_{0}^{s_{n-1}} d s_{n} \tau_{i s_{n}}(V) \ldots \tau_{i s_{1}}(V)
$$

Let $U_{V}(t)=\exp \left\{i t H_{v}\right\}$ be the unitary group which is implemented by $\tau_{i}^{V}$ in the cyclic GNS-representation $\left(\mathscr{H}_{\omega_{V}}, \pi_{\omega_{V}}, \Omega_{\omega_{V}}\right)$ with respect to $\omega_{V}(V$ can be equal to 0 here).

Corollary. Under the conditions of Theorem $2, H_{0}$ and $H_{V}$ are unitary equivalent.

Note. The same technique works for the case $(n \geqq 1, v \geqq 3)$

$$
V=\varepsilon \sum_{n=1}^{N} \sum_{x_{1} \in \mathbb{Z}^{v}} V_{n}\left(x_{1}, \ldots, x_{n}\right)\left(\prod_{i=1}^{n} \chi_{\Omega}\left(x_{i}\right)\right) a^{*}\left(x_{1}\right) \ldots a^{*}\left(x_{n}\right) a\left(x_{n}\right) \ldots a\left(x_{1}\right) .
$$

The case $n=1$ is explicitly solvable. It corresponds to the free Fermi-gas in the exterior field $V(x)$. In this case one finds examples for dimensions $v=1,2$ where bounded states appear [6].

\section{Existence of Möller Morphisms}

We shall prove Theorem 1 here. We begin with the case $v \geqq 3$. The cases $v=1,2$ are slightly different. The proof is a simple modification of similar proofs in [1-3]. It is sufficient (see Theorem 4 in [1]) to prove that $(\mathfrak{A}, \tau)$ is asymptotically integrable with respect to $V$, i.e. one must specify a dense subset $\hat{\mathfrak{A}} \subset \mathfrak{A}$ such that for any $A \in \hat{\mathfrak{U}}$ the function $\left\|\left[\tau_{t}(V), A\right]\right\|$ is absolutely integrable in $t$. We put

$$
\begin{aligned}
\hat{\mathfrak{U}}=\{ & \left\{a^{*}\left(f_{1}\right) a^{*}\left(f_{2}\right) \ldots a^{*}\left(f_{m}\right) a\left(g_{1}\right) a\left(g_{2}\right) \ldots a\left(g_{n}\right), m \geqq 0, n \geqq 0, f_{i}, g_{i}\right. \\
& \text { are local, i.e. have finite supports in } \left.\mathbb{Z}^{\nu}\right\} .
\end{aligned}
$$

Let us put $A=a(f), f$ being local, $(\cdot, \cdot)$ is the scalar product in $K=l_{2}\left(\mathbb{Z}^{v}\right)$. One has

$$
\begin{aligned}
& \left\|\left[\tau_{t}(A), V\right]\right\| \\
& =\left\|\left[a\left(e^{i t H} f\right), V\right]\right\|=\left\|\varepsilon \sum_{x, y \in \mathbb{Z}^{v}} V_{\Omega}(x, y)\left[a\left(e^{i t H} f\right), a_{x}^{*} a_{y}^{*} a_{y} a_{x}\right]\right\| \\
& =\| \varepsilon \sum_{x, y \in \mathbb{Z}^{v}} V_{\Omega}(x, y)\left(-a_{x}^{*} a\left(e^{i t H} f\right) a_{y}^{*} a_{y} a_{x}\right. \\
& \left.\quad+\left(e^{i t H} f, \delta_{x}\right) a_{y}^{*} a_{y} a_{x}-a_{x}^{*} a_{y}^{*} a_{y} a_{x} a\left(e^{i t H} f\right)\right) \| \\
& \leqq|\varepsilon| \sum_{x, y \in \Omega} \mid V_{\Omega}(x, y) \|\left(\left|\left(e^{i t H} f, \delta_{x}\right)\right|+\left|\left(e^{i t H} f, \delta_{y}\right)\right|\right) .
\end{aligned}
$$


There exist $C(f)>0$ such that uniformly in $z \in \Omega$

$$
\left|\left(e^{i t H} f, \delta_{z}\right)\right| \leqq C(f) \frac{1}{(1+|t|)^{v / 2}}
$$

(see Appendix A). It follows from (2.1) and (2.2) that $\left\|\left[\tau_{t}(A), V\right]\right\|$ is asymptotically integrable for $v \geqq 3$.

The case $A=a^{*}(f)$ can be treated similarly. As $\tau_{-t}^{V} \tau_{t}$ is the homomorphism of $\mathfrak{U}$ into itself the operators $\gamma_{ \pm}(A)$ exist for any $A \in \hat{\mathfrak{A}}$.

Let us consider the case $v=1$. We choose

$$
\begin{aligned}
& \hat{\mathfrak{U}}=\left\{a^{*}\left(f_{1}\right) a^{*}\left(f_{2}\right) \ldots a^{*}\left(f_{m}\right) a\left(g_{1}\right) a\left(g_{2}\right) \ldots a\left(g_{n}\right),\right. \\
&\left.m, n \geqq 0, f_{i}, g_{i} \in K_{0}\right\},
\end{aligned}
$$

where

$$
\begin{gathered}
K_{0}=\left\{f: \tilde{f} \in L_{2}([0,2 \pi]), \tilde{f}(0)=\tilde{f}(\pi)=\tilde{f}(2 \pi)\right. \\
\left.=\tilde{f}^{\prime}(0)=\tilde{f}^{\prime}(\pi)=\tilde{f}^{\prime}(2 \pi)=0\right\} .
\end{gathered}
$$

It is evident that $\hat{\mathfrak{A}}$ is also dense in $\mathfrak{A}$. We have for $f \in K_{0}$

$$
\begin{aligned}
\left|\left(e^{i t H} f, \delta_{z}\right)\right| & =\left|\frac{1}{\sqrt{2 \pi}} \int_{0}^{2 \pi} e^{-2 i t(1-\cos \varphi)+i t \mu} \tilde{\tilde{f}} e^{i z \varphi} d \varphi\right| \\
& =\left|\frac{1}{2 t \sqrt{2 \pi}} \int_{0}^{2 \pi} \frac{\tilde{\tilde{f}}(\varphi) e^{i z \varphi}}{\sin \varphi} \mathrm{d}\left(\mathrm{e}^{-2 i t(1-\cos \varphi)}\right)\right| .
\end{aligned}
$$

Let us put

$$
g_{z}(\varphi)=\frac{\tilde{\tilde{f}}(\varphi)}{\sin \varphi} e^{i z \varphi}
$$

Integrating by parts the integral in the right-hand side of (2.5) and using again Appendix A in the similar way we prove Theorem 1 for $v=1$.

For $v=2$ one can choose

$$
\begin{aligned}
\hat{\mathfrak{U}}= & \left\{a^{*}\left(f_{1}\right) a^{*}\left(f_{2}\right) \ldots a^{*}\left(f_{m}\right) a\left(g_{1}\right) a\left(g_{2}\right) \ldots a\left(g_{n}\right),\right. \\
& \left.m, n \geqq 0, f, g \in K_{0} \otimes K_{0}\right\} .
\end{aligned}
$$

\section{Invertibility of Möller Morphisms}

We shall prove here Theorem 2. As in Sect. 2, in order to prove the existence of $\bar{\gamma}_{ \pm}$ we must prove that for any $A \in \hat{\mathrm{g}}$, $\hat{\mathfrak{U}}$ being dense in $\mathfrak{A}, R_{A}(t)=\left\|\left[\tau_{-t}^{V}(V), A\right]\right\|$ is asymptotically integrable in $t$. Indeed, one can derive Robinson's equation $[1,3]$ for this case

$$
\frac{d}{d t} \tau_{-t} \tau_{t}^{V}(A)=i \tau_{-t} \tau_{t}^{V}\left(\left[\tau_{-t}^{V}(V), A\right]\right)
$$


(It follows from the explicit calculations in finite volumes.) Again we take

$$
\begin{aligned}
\hat{\mathfrak{A}}= & \left\{a^{*}\left(f_{1}\right) a^{*}\left(f_{2}\right) \ldots a^{*}\left(f_{m}\right) a\left(g_{1}\right) a\left(g_{2}\right) \ldots a\left(g_{n}\right),\right. \\
& \left.m, n \geqq 0, f_{i}, g_{i} \text { are local }\right\} .
\end{aligned}
$$

In order to prove the existence of $\bar{\gamma}_{-}$one must show that

$$
\int_{0}^{\infty} R_{a}^{\#(f)}(t) d t<\infty
$$

for any local $f, a^{\#}(f)=a(f)$ or $a^{\#}(f)=a^{*}(f)$.

We have [1]

$$
\begin{aligned}
& \int_{0}^{\infty} R_{a}^{\#}(f) d t=\int_{0}^{\infty}\left\|\left[a^{\#}(f), \tau_{t}(V)\right]\right\| d t \\
& +\| \sum_{n=1}^{\infty} i^{n} \underset{0 \leqq s_{1} \leqq s_{2} \leqq \ldots \leqq s_{n} \leqq t<\infty}{\int \ldots \int} d s_{1} \ldots d s_{n} d t \\
& \cdot\left[a^{\#}(f),\left[\tau_{s_{1}}(V), \ldots,\left[\tau_{s_{n}}(V), \tau_{t}(V)\right] \ldots\right]\right] \|
\end{aligned}
$$

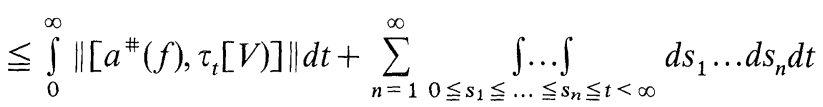

$$
\begin{aligned}
& \cdot\left\|\left[a^{\#}(f),\left[\tau_{s_{1}}(V), \ldots,\left[\tau_{s_{n}}(V), \tau_{t}(V)\right] \ldots\right]\right]\right\| .
\end{aligned}
$$

Theorem 3. Under the conditions of Theorem 2 there exist constants $C_{1}=C(V, \Omega)$ $>0$, not depending on $f$ and $C(f)>0$ such that for any $n \in \mathbb{N}$

a)

$$
\begin{gathered}
\int_{0}^{\infty}\left\|\left[\tau_{t}(V), a^{\#}(f)\right]\right\| d t \leqq|\varepsilon| C_{1} C(f), \\
\text { b) } \int_{0 \leqq s_{1} \leqq \ldots \leqq s_{n} \leqq t<\infty}\left\|\left[a^{\#}(f),\left[\tau_{s_{1}}(V), \ldots,\left[\tau_{s_{n}}(V), \tau_{t}(V)\right] \ldots\right]\right]\right\| d s_{1} \ldots d s_{n} d t \\
\leqq C(f) C_{1}^{n}|\varepsilon|^{n+1} .
\end{gathered}
$$

It is evident that (3.4) implies the absolute convergence of (3.2) for $|\varepsilon|<\frac{1}{C_{1}}$.

Proof. Let $f_{1}, f_{2}, h_{i} \in l_{2}\left(\mathbb{Z}^{v}\right), i=1, \ldots, k,\left(f_{1} \rightarrow, f_{2}\right)=0$.

Then the following formula holds:

$$
\begin{aligned}
& {\left[a^{*}\left(f_{1}\right) a^{*}\left(f_{2}\right) a(f) a\left(f_{1}\right), a^{\#}\left(h_{1}\right) \ldots a^{\#}\left(h_{k}\right)\right.} \\
& =-\sum_{i=1}^{k} \chi(\#(i)) \widehat{\left(f_{1}, h_{i}\right)} a^{\#}\left(h_{1}\right) \ldots a^{\#}\left(h_{i-1}\right) a^{\#(i)}\left(f_{1}\right) a^{*}\left(f_{2}\right) a\left(f_{2}\right) \\
& \cdot a^{*}\left(h_{i+1}\right) \ldots a^{\#}\left(h_{k}\right)+\sum_{i=1}^{k} \chi(\#(i)) \widehat{\left(f_{2}, h_{i}\right)} a^{\#}\left(h_{1}\right) \ldots a^{\#}\left(h_{i-1}\right) \\
& \cdot a^{*}\left(f_{1}\right) a^{\#(i)}\left(f_{2}\right) a\left(f_{1}\right) a^{\#}\left(h_{i+1}\right) \ldots a^{\#}\left(h_{k}\right),
\end{aligned}
$$


where

$$
\begin{gathered}
\#(i)= \begin{cases}*, & \text { if } \quad a^{\#}\left(h_{i}\right)=a^{*}\left(h_{i}\right) \\
\text { absence of } *, & \text { if } \quad a^{\#}\left(h_{i}\right)=a\left(h_{i}\right),\end{cases} \\
\chi(x)=-1, \quad \chi(\text { absence of } *)=1, \\
\widehat{(f, h)}=\left\{\begin{array}{ll}
\frac{(f, h),}{(f, h),} & \#(h)=*
\end{array}, \text { absence of } * .\right.
\end{gathered}
$$

We use simple identities

$$
\begin{aligned}
& a^{*}\left(f_{1}\right) a^{*}\left(f_{2}\right) a\left(f_{2}\right) a\left(f_{1}\right) a(h) \\
&= a(h) a^{*}\left(f_{1}\right) a^{*}\left(f_{2}\right) a\left(f_{2}\right) a\left(f_{1}\right)-\left(h, f_{1}\right) a^{*}\left(f_{2}\right) a\left(f_{2}\right) a\left(f_{1}\right) \\
&+\left(h, f_{2}\right) a^{*}\left(f_{1}\right) a\left(f_{2}\right) a\left(f_{1}\right), a^{*}\left(f_{1}\right) a^{*}\left(f_{2}\right) a\left(f_{2}\right) a\left(f_{1}\right) a^{*}(h) \\
&= a^{*}(h) a^{*}\left(f_{1}\right) a^{*}\left(f_{2}\right) a\left(f_{2}\right) a\left(f_{1}\right)+\left(f_{1}, h\right) a^{*}\left(f_{1}\right) a^{*}\left(f_{2}\right) a\left(f_{2}\right) \\
&-\left(f_{2}, h\right) a^{*}\left(f_{1}\right) a^{*}\left(f_{2}\right) a\left(f_{1}\right),
\end{aligned}
$$

and consider now

$$
a^{*}\left(f_{1}\right) a^{*}\left(f_{2}\right) a\left(f_{2}\right) a\left(f_{1}\right) a^{\#}\left(h_{1}\right) \ldots a^{\#}\left(h_{k}\right) .
$$

Using (3.7) we drag through to the left all $a^{\#}\left(h_{1}\right), \ldots, a^{\#}\left(h_{k}\right)$ in (3.8) thus proving

Let us put

$$
a_{x}^{\#}(s) \stackrel{\text { def }}{=} a\left(e^{i s H} \delta_{x}\right) .
$$

Lemma. The following estimate holds $\left(t=s_{n+1}\right)$

$$
\begin{aligned}
& \|\left[a^{\#}(f),\left[a_{x_{1}}^{*}\left(s_{1}\right) a_{y_{1}}^{*}\left(s_{1}\right) a_{y_{1}}\left(s_{1}\right) a_{x_{1}}\left(s_{1}\right), \ldots,\left[a_{x_{n}}^{*}\left(s_{n}\right) a_{y_{n}}^{*}\left(s_{n}\right) a_{y_{n}}\left(s_{n}\right) a_{x_{n}}\left(s_{n}\right),\right.\right.\right. \\
&\left.\left.\left.\quad \cdot a_{x_{n+1}}^{*}\left(s_{n+1}\right) a_{y_{n+1}}^{*}\left(s_{n+1}\right) a_{y_{n+1}}\left(s_{n+1}\right) a_{x_{n+1}}\left(s_{n+1}\right)\right] \ldots\right]\right] \| \\
& \leqq 2^{n} \sum_{j_{0}, j_{1}, \ldots, j_{n}\left\{z_{z}\right\},\left\{z_{1}^{\prime}\right\}} \mid\left(f, e^{i s_{J_{0}} H} \delta_{\left.z_{j_{0}}\right)} \mid\right. \\
& \cdot\left|\left(e^{i s_{1} H} \delta_{z_{1}}, e^{i s_{J_{1}} H} \delta_{z_{j_{1}}^{\prime}}\right)\right| \ldots\left|\left(e^{i s_{n-1} H} \delta_{z_{n-1}}, e^{i s_{j_{n-1}} H} \delta_{z_{j_{n-1}}}\right)\right| \\
& \cdot\left|\left(e^{i s_{n} H} \delta_{z_{n}}, e^{i s_{n+1} H} \delta_{z_{n+1}^{\prime}}\right)\right|
\end{aligned}
$$

where the sum $\sum_{\left\{z_{i}\right\},\left\{z_{i}^{\prime}\right\}}$ si taken over all $2^{2 n+3}$ ordered sequences $z_{i}=x_{i}$ or $y_{i}, z_{i}^{\prime}=x_{i}$ or $y_{i}$, the sum $\sum_{j_{0}, \ldots, j_{n}}$ is over all sequences $\left(j_{0}, j_{1}, \ldots, j_{n}\right)$ such that for all $k 0 \leqq k<j_{k}$ $\leqq n+1$ for any $l, 0 \leqq l \leqq n+1, j_{i}$ can be equal to $l$ nor more than for four values of $i$.

Proof. It is convenient to use diagrams (graphs). The vertices of a diagram are the points $s_{0}=0, s_{1}, \ldots, s_{n}, s_{n+1}$ on the real line. The lines are the pairs $\left(s_{0}, s_{j_{0}}\right),\left(s_{1}\right.$, $\left.s_{j_{1}}\right), \ldots,\left(s_{n-1}, s_{j_{n-1}}\right),\left(s_{n}, s_{n+1}\right)$ which enter in the right-hand side of (3.9):

a) the diagrams are connected;

b) the number of edges incident to any vertex does not exceed $k=4$ (if $s_{0} \leqq s_{1}$ $\left.\leqq s_{2} \leqq \ldots \leqq s_{n+1}\right)$. 
To prove (3.9) we use formula (3.6) inductively. From the first commutator we obtain one edge of the diagram, from the second one we obtain the other edge, and so on. We have two $2^{3 n}$ terms with the same diagrams. The norm of the remaining operation and annihilation operators is bounded by 1 . that

Due to Appendix A there exist constants $C=C(V, \Omega)>0$ and $C_{2}(f)>0$ such

$$
\begin{gathered}
\left|\left(f, e^{i s H} \delta_{z}\right)\right| \leqq C_{2}(f) \frac{1}{(1+|s|)^{v / 2}} \\
\left(\delta_{z_{1}}, e^{i s H} \delta_{z_{2}}\right) \leqq C \frac{1}{(1+|s|)^{v / 2}}
\end{gathered}
$$

for all $z, z_{1}, z_{2} \in \Omega$.

Then the upper bound of the right-hand side of (3.9) takes the form

$$
\begin{gathered}
2^{3 n+1} C^{n} C_{2}(f) \sum_{j_{0}, j_{1}, \ldots, j_{n}} \frac{1}{\left(1+\left|s_{j_{0}}\right|\right)^{v / 2}} \frac{1}{\left(1+\left|s_{j_{1}}-s_{1}\right|\right)^{v / 2}} \cdots \\
\cdots \frac{1}{\left(1+\left|s_{j_{n-1}}-s_{n-1}\right|\right)^{v / 2}} \frac{1}{\left(1+\left|s_{j_{n}}-s_{n}\right|\right)^{v / 2}} .
\end{gathered}
$$

\section{The Proof of the Main Estimate}

We prove (3.4) here, i.e.

$$
\begin{gathered}
\sum_{j_{0}, j_{1}, \ldots, j_{n} 0 \leqq s_{1} \leqq \ldots \leqq s_{n+1}<\infty} \frac{1}{\left(1+\left|s_{j_{0}}\right|\right)^{v / 2}} \frac{1}{\left(1+\left|s_{j_{1}}-s_{1}\right|\right)^{v / 2}} \ldots \\
\ldots \frac{1}{\left(1+\left|s_{j_{n}}-s_{n}\right|\right)^{v / 2}} d s_{1} \ldots d s_{n+1} \leqq C^{n}\left(\int_{-\infty}^{\infty} \frac{1}{(1+|s|)^{v / 2}} d s\right)^{n+1}
\end{gathered}
$$

where $C$ does not depend on $n$.

We approximate both sides of (4.1) by Riemannian sums

$$
\begin{gathered}
\sum_{j_{0}, \ldots, j_{n}} \delta^{n+1} \sum_{0<s_{1}<\ldots<s_{n+1}<\infty} \frac{1}{\left(1+\left|s_{j_{0}}\right|\right)^{v / 2}} \frac{1}{\left(1+\left|s_{j_{1}}-s_{1}\right|\right)^{v / 2}} \cdots \\
\ldots \frac{1}{\left(1+\left|s_{j_{n}}-s_{n}\right|\right)^{v / 2}} \leqq C^{n} \delta^{n+1}\left(\sum_{r \neq 0} \frac{1}{(1+|r|)^{v / 2}}\right)^{n+1} \\
=C^{n} \delta^{n+1} \sum_{r_{1} \neq 0} \ldots \sum_{r_{n+1} \neq 0} \frac{1}{\left(1+\left|r_{1}\right|\right)^{v / 2}} \cdots \frac{1}{\left(1+\left|r_{n+1}\right|\right)^{v / 2}} .
\end{gathered}
$$

In both sides $s_{i}, r_{i} \in \mathbb{Z}_{\delta}$ (one-dimensional $\delta$-lattice). The sum in the left-hand side of (4.2) is dominated by the sum over all admissible diagrams [with the properties a) and b)] whose vertices are numbered as $0<s_{1}<s_{2}<\ldots<s_{n+1}$. We denote this sum by $\sum_{G} I_{G}$, where $I_{G}$ is the contribution of the corresponding diagram. The right- 
hand sum $\sum^{(r)}$ is taken over all arrays $\left(r_{1}, \ldots, r_{n+1}\right)$ of edges with the nonzero lengths $r_{1}, \ldots, r_{n+1}$.

We omit $\delta^{n+1}$ and want to prove that

$$
\sum_{G} I_{G} \leqq C^{n} \sum^{(r)}
$$

To prove (4.3) we use the argument similar to the one used in Sect. 1.5 of [7].

Given an array $\left(r_{1}, \ldots, r_{n+1}\right)$ we define the algorithm by which one can construct not more than $C^{n}$ admissible diagrams with the contribution equal to

$$
\frac{1}{\left(1+\left|r_{1}\right|\right)^{v / 2}} \cdots \frac{1}{\left(1+\left|r_{n+1}\right|\right)^{v / 2}} .
$$

The algorithm consists of not more than $4(n+2)$ steps. We numerate these steps by

$$
(0,1), \ldots,(0,4),(1,1), \ldots,(1,4), \ldots,(n+1,1), \ldots,(n+1,4) \text {. }
$$

On the step $(0,1)$ we take $r_{1}$ and construct an edge from 0 to $r_{1}$. We construct vertices $0, r_{1}$ and the line between them. Then we proceed by induction. The lines of length $r_{1}, \ldots, r_{k}$ are already constructed and we are on the step $(i, j)$. The rules of the algorithm are the following:

1. On each step we decide whether to construct 0 or 1 line (and so 0 or 1 new vertex).

2. If on the step $(i, j)$ we decided not to construct a line then on the steps $\left(i, j^{\prime}\right)$, $j<j^{\prime}$ we also do not construct lines.

3. On the step $(i, 1)$ we choose one of the already constructed vertices $V_{i}$ and on all steps $(i, 1), \ldots,(i, 4)$ we can draw lines only from $V_{i}$. We call $V_{i}$ "used on the step of $i$."

4. The choice of $V_{i}$ is uniquely defined by the rule: $V_{i}$ is the first already constructed vertex not used in earlier steps.

5 . The algorithm stops either on the step $(n+1,4)$ either when there are no nonused vertices or when $(n+1)$-lines are constructed (i.e. all $r_{1}, \ldots, r_{n+1}$ are exhausted).

It is evident that each $G$ will be constructed and each array $\left(r_{1}, \ldots, r_{n+1}\right)$ is used not more than $\left(5 \cdot 2^{4}\right)^{n+1}$ times.

\section{Unitary Equivalence}

We shall prove Corollary 1 here. As $\omega_{0}$ is the unique $\left(\tau_{t, \beta}\right)$-KMS state and $\omega_{V}$ is the unique $\left(\tau_{t, \beta}^{V}\right)$-KMS state [3], then by Theorem 2 of [1] (see also [3]) we have

$$
\omega_{ \pm}(A) \stackrel{\text { def }}{=} \omega_{V}\left(\gamma_{ \pm}(A)\right) \equiv \omega_{0}(A)
$$

for all $A \in \mathfrak{A}$. Let us define the operators $U_{ \pm}: \mathscr{H}_{\omega_{0}} \rightarrow \mathscr{H}_{\omega_{V}}$ by

$$
U_{ \pm}\left(\pi_{\omega_{0}}(A) \Omega_{\omega_{0}}\right)=\pi_{\omega_{V}}\left(\gamma_{ \pm}(A)\right) \Omega_{\omega_{V}} .
$$


They are isometric

$$
\begin{aligned}
& \left(U_{ \pm}\left(\pi_{\omega_{0}}(A) \Omega_{\omega_{0}}\right), U_{ \pm}\left(\pi_{\omega_{0}}(B) \Omega_{\omega_{0}}\right)\right) \\
& \quad=\omega_{V}\left(\left(\gamma_{ \pm}(B)\right)^{*} \gamma_{ \pm}(A)\right)=\omega_{V}\left(\gamma_{ \pm}\left(B^{*} A\right)\right) \\
& \quad=\omega_{0}\left(B^{*} A\right)=\left(\pi_{\omega_{0}}(A) \Omega_{\omega_{0}}, \pi_{\omega_{0}}(B) \Omega_{\omega_{0}}\right)
\end{aligned}
$$

and unitary

$$
U_{ \pm}^{-1}\left(\pi_{\omega_{V}}(A) \Omega_{\omega_{V}}\right)=\pi_{\omega_{0}}\left(\gamma_{ \pm}^{-1}(A)\right) \Omega_{\omega_{0}}
$$

We have

$$
\tau_{t}^{V}=\gamma_{ \pm} \tau_{t} \gamma_{ \pm}^{-1}
$$

By definition

$$
\begin{aligned}
e^{i t H_{0}}\left(\pi_{\omega_{0}}(A) \Omega_{\omega_{0}}\right) & =\pi_{\omega_{0}}\left(\tau_{t}(A)\right) \Omega_{\omega_{0}}, \\
e^{i t H_{V}}\left(\pi_{\omega_{V}}(A) \Omega_{\omega_{V}}\right) & =\pi_{\omega_{V}}\left(\tau_{t}^{V}(A)\right) \Omega_{\omega_{V}} .
\end{aligned}
$$

It follows that

$$
\begin{aligned}
& U_{ \pm} e^{i t H_{0}} U_{ \pm}^{-1}\left(\pi_{\omega_{0}}(A) \Omega_{\omega_{0}}\right) \\
& \quad=U_{ \pm} e^{i t H_{0}}\left(\pi_{\omega_{0}}\left(\gamma_{ \pm}^{-1}(A)\right) \Omega_{\omega_{0}}\right)=U_{ \pm}\left(\pi_{\omega_{0}}\left(\tau_{t} \gamma_{ \pm}^{-1}(A)\right) \Omega_{\omega_{0}}\right) \\
& \quad=\pi_{\omega_{V}}\left(\tau_{t}^{V}(A)\right) \Omega_{\omega_{V}}=e^{i t H_{V}}\left(\pi_{\omega_{V}}(A) \Omega_{\omega_{V}}\right),
\end{aligned}
$$

and so

$$
U_{ \pm} H_{0} U_{ \pm}^{-1}=H_{V}
$$

Corollary. Under the conditions of Theorem 2 the spectrum of $H_{V}$ (except nondegenerate eigenvalue 0) is absolutely-continuous.

This follows from Corollary 1 and Appendix B.

\section{Appendix A}

Let $f$ be infinitely differentiable on the $v$-dimensional torus $T, v \geqq 1$, and $\Omega$ is a finite subset of $\mathbb{Z}^{v}$. Then there exist $C=C(f, \Omega)>0$ such that

$$
\left|\int_{T^{v}} \exp \left[i t\left(\sum_{j=1}^{v} 2\left(1-\cos \varphi_{j}\right)\right)\right] f(\varphi) e^{i(x, \varphi)} d \varphi\right| \leqq C \frac{1}{(1+|t|)^{v / 2}}
$$

for all $x \in \Omega, \varphi=\left(\varphi_{1}, \ldots, \varphi_{\nu}\right)$.

We must only note that for fixed $x \in \Omega$ (A.1) follows from the stationary phase method.

From (A.1) it follows evidently that there exists $C=C(\Omega)$ such that

$$
\left|\int_{T^{v}} \exp \left[i t\left(\sum_{j=1}^{v} 2\left(1-\cos \varphi_{j}\right)\right)\right] e^{i(x-y, \varphi)} d \varphi\right| \leqq C \frac{1}{(1+|t|)^{v / 2}}
$$

for all $x, y \in \Omega$. 


\section{Appendix B}

We obtain here the spectral decomposition of $H_{0}$ (we follow [4]).

Definition 1. The state $\omega$ on the CAR-algebra $\mathfrak{A}$ over $K$ is called (gauge-invariant) quasi-free state iff

$$
\omega\left(a^{*}\left(f_{m}\right) \ldots a^{*}\left(f_{1}\right) a\left(g_{1}\right) \ldots a\left(g_{n}\right)\right)=\delta_{m n} \operatorname{det}\left(\left(g_{i}, B f_{j}\right)\right)
$$

for all $f_{i}, g_{j} \in K$, where $B$ is the linear operator in $K$ such that $0 \leqq B \leqq 1_{K}[5]$.

Definition 2. The Wick monomial is the following polinomial $\left(::_{\omega} \stackrel{\text { def }}{=}::\right)$

$$
\begin{aligned}
& : a^{*}\left(f_{1}\right) \ldots a^{*}\left(f_{m}\right) a\left(f_{m+1}\right) \ldots a\left(f_{m+n}\right): \\
& =\sum_{k=0}^{\min (m, n)}(-1)^{k} \sum_{\sigma} \operatorname{sgn} \sigma \prod_{l=1}^{k} \omega\left(a^{*}\left(f_{i_{l}}\right) a\left(f_{j_{l}}\right)\right) \\
& \quad \cdot a^{*}\left(f_{1}\right) \ldots a^{*} \widehat{\left(f_{i_{1}}\right)} \ldots a^{*}\left(\widehat{f_{i_{k}}}\right) \ldots a^{*}\left(f_{m}\right) a\left(f_{m+1}\right) \ldots a\left(\widehat{f_{j_{s}}}\right) \ldots a\left(f_{m+n}\right),
\end{aligned}
$$

where $\sum_{\sigma}$ is the sum over all permutations $\sigma \in S_{m+n}$ such that

$$
\sigma=\left(\begin{array}{llll}
1 & 2 \ldots 2 k-1 & 2 k & 2 k+1 \ldots m+n \\
i_{1} & j_{1} \ldots i_{k} & j_{k} & r_{1} \ldots r_{m+n-2 k}
\end{array}\right)
$$

for any sequence $1 \leqq i_{1}<i_{2}<\ldots<i_{k} \leqq m, m+1 \leqq j_{l} \leqq m+n, l=1, \ldots, k$ and any increasing sequence $r_{1}, \ldots, r_{m+n-2 k}$ of numbers $1,2, \ldots, m+n$ different from $i_{1}, \ldots, i_{k}$, $j_{1}, \ldots, j_{k}$.

Lemma 1 (The properties of Wick monomials). Let $\omega$ be a quasi-free state. Then

$$
\begin{aligned}
& \quad\left(: a^{*}\left(f_{1}\right) \ldots a^{*}\left(f_{m}\right) a\left(g_{1}\right) \ldots a\left(g_{n}\right):\right)^{*} \\
& \quad=: a^{*}\left(g_{n}\right) \ldots a^{*}\left(g_{1}\right) a\left(f_{m}\right) \ldots a\left(f_{1}\right): \\
& \omega\left(: a^{*}\left(f_{1}\right) \ldots a^{*}\left(f_{n}\right) a\left(g_{1}\right) \ldots a\left(g_{m}\right):\right)=0 \quad \text { if } m+n>0, \\
& \omega\left(: a^{*}\left(f_{m}\right) \ldots a^{*}\left(f_{1}\right) a\left(g_{1}\right) \ldots a\left(g_{n}\right):: a^{*}\left(h_{k}\right) \ldots a^{*}\left(h_{1}\right)\right. \\
& \left.\cdot a\left(u_{1}\right) \ldots a\left(u_{l}\right):\right)=\omega\left(a^{*}\left(f_{m}\right) \ldots a^{*}\left(f_{1}\right) a\left(u_{1}\right) \ldots a\left(u_{l}\right)\right), \\
& \omega\left(a\left(g_{1}\right) \ldots a\left(g_{n}\right) a^{*}\left(h_{k}\right) \ldots a^{*}\left(h_{1}\right)\right) \\
& =\delta_{n k} \delta_{m l} \operatorname{det}\left(\left(u_{i}, B f_{j}\right)\right) \operatorname{det}\left(\left(g_{i},(I-B) h_{j}\right) .\right.
\end{aligned}
$$

Proof of 1) is evident. For a proof of 2) and 3), see [4].

Definition 3. Let $e^{i t H}$ be a unitary group in $K$. The group $\tau_{t}$ of automorphisms of $\mathfrak{A}$ induced by

$$
\tau_{t}(a(f))=a\left(e^{i t H} f\right), \quad \tau_{t}\left(a^{*}(f)\right)=a^{*}\left(e^{i t H} f\right)
$$

will be called the free dynamics. 
Let $\left(\mathscr{H}_{\omega}, \pi_{\omega}, \Omega_{\omega}\right)$ be the cyclic GNS-representation with respect to quasi-free $\omega$. We define $H_{\omega}$ by

$$
\begin{gathered}
e^{i t H_{\omega}}\left(\bar{\pi}_{\omega}(A)\right)=\bar{\pi}_{\omega}\left(\tau_{t}(A)\right), \\
\bar{\pi}_{\omega}(A) \stackrel{\text { def }}{=} \pi_{\omega}(A) \Omega_{\omega} .
\end{gathered}
$$

Lemma 2. Let $B$ and $H$ commute. Then the subspaces

$$
\mathscr{H}_{m, n}=\left\{\bar{\pi}_{\omega}\left(: a^{*}\left(f_{1}\right) \ldots a^{*}\left(f_{m}\right) a\left(g_{1}\right) \ldots a\left(g_{n}\right):\right), f_{i}, g_{i} \in K\right\}, \quad \mathscr{H}_{0,0}=\left\{\Omega_{\omega}\right\}
$$

are mutually orthogonal, invariant with respect to $H_{\omega}$ and

$$
\mathscr{H}_{\omega}=\bigoplus_{m, n=0}^{\infty} \mathscr{H}_{m, n} .
$$

Proof. Orthogonality follows from properties 2) and 3) of Wick monomials. Invariance follows as

$$
\begin{aligned}
\omega\left(a^{*}\left(e^{i t H} f\right) a\left(e^{i t H} g\right)\right) & =\left(e^{i t H} g, B e^{i t H} f\right) \\
& =\left(e^{i t H} g, e^{i t H} B f\right)=(g, B f)=\omega\left(a^{*}(f) a(g)\right)
\end{aligned}
$$

implies

$$
\tau_{t}\left(: a^{*}\left(f_{1}\right) \ldots a^{*}\left(f_{m}\right) a\left(g_{1}\right) \ldots a\left(g_{n}\right):\right)=: \tau_{t}\left(a^{*}\left(f_{1}\right) \ldots a^{*}\left(f_{m}\right) a\left(g_{1}\right) \ldots a\left(g_{n}\right)\right): .
$$

Further we shall consider only a Fermi-gas on a lattice. We recall (see [3]) that in this case

$$
\begin{gathered}
K=l_{2}\left(\mathbb{Z}^{v}\right), \quad H=-\Delta+\mu-1_{K}, \\
B=\exp (-\beta H)\left(1_{K}+\exp (-\beta H)\right)^{-1}, \quad 0<\beta<\infty .
\end{gathered}
$$

Let us put

$$
\begin{aligned}
& \hat{\mathscr{H}}_{m, n} \stackrel{\text { def }}{=} {\left.[\underbrace{(K \otimes \ldots \otimes K}_{m})^{a s}\right] \otimes\left[(\underbrace{\tilde{K} \otimes \ldots \otimes \tilde{K}}_{n})^{a s}\right], } \\
&\left(\tilde{K},(\cdot, \cdot)_{\tilde{K}}\right) \stackrel{\text { def }}{=}\left(K, \overline{\left.(\cdot, \cdot)_{K}\right)} .\right.
\end{aligned}
$$

Theorem 4. There exists the unitary operator

$$
U_{m, n}: \mathscr{H}_{m, n} \rightarrow \hat{\mathscr{H}}_{m, n}
$$

such that

$$
U_{m, n} H_{\omega} U_{m, n}^{-1} \equiv \Delta_{1}+\ldots+\Delta_{m}-\Delta_{m+1}-\ldots-\Delta_{m+n}+\mu(n-m) .
$$

In other words $\mathscr{H}_{\omega}$ is isomorphic to the tensor product $\mathscr{F}_{-} \otimes \tilde{\mathscr{F}}_{-}$of the Fock space $\mathscr{F}_{-}$and "anti Fock space" $\tilde{\mathscr{F}}$, and $\Gamma\left(e^{i t H}\right)$ is the second quantities of $e^{i t H}$ and

$$
e^{i t H_{\omega}} \sim \Gamma\left(e^{i t H}\right) \otimes \Gamma\left(e^{-i t H}\right) .
$$


The proof is easily obtained if we put

$$
\begin{aligned}
& U_{m, n} \bar{\pi}_{\omega}\left(: a^{*}\left(f_{1}\right) \ldots a^{*}\left(f_{m}\right) a\left(g_{1}\right) \ldots a\left(g_{n}\right):\right) \\
& \stackrel{\text { def }}{=}\left(\left(1_{K}-B\right)^{1 / 2} f_{1} \otimes \ldots \otimes\left(1_{K}-B\right)^{1 / 2} f_{m}\right)^{a s} \\
& \otimes\left(B^{1 / 2} g_{1} \otimes \ldots \otimes B^{1 / 2} g_{n}\right)^{a s} .
\end{aligned}
$$

Let us note that $\operatorname{Im} U_{m, n}=\hat{\mathscr{H}}_{m, n}$ as $B^{1 / 2}$ and $\left(1_{K}-B\right)^{1 / 2}$ are invertible.

Corollary (Fourier representation). $\mathscr{H}_{\omega}$ is isomorphic to $L_{2}^{a s}\left(T^{v m}\right) \otimes \tilde{L}_{2}^{a s}\left(T^{v n}\right)$. Under this isomorphism $\left.H_{\omega}\right|_{\mathscr{H}_{m, n}}$ is unitary equivalent to the multiplication onto the function $\left(\varphi_{j}^{(k)} \in[0,2 \pi]\right)$

$$
\left(-\sum_{j=1}^{m} \sum_{k=1}^{\nu} 2\left(1-\cos \varphi_{j}^{(k)}\right)+\sum_{j=m+1}^{m+n} \sum_{k=1}^{\nu} 2\left(1-\cos \varphi_{j}^{(k)}\right)+\mu(n-m)\right) .
$$

Thus the spectrum on $\mathscr{H}_{\omega_{0}} \ominus \mathscr{H}_{0,0}$ is absolutely-continuous.

\section{References}

1. Robinson, D.W.: Return to equilibrium. Commun. Math. Phys. 31, 171-189 (1973)

2. Evans, D.E.: Scattering in CAR-algebra. Commun. Math. Phys. 48, 23-30 (1976)

3. Bratteli, O., Robinson, D.W.: Operator algebras and quantum statistical mechanics. II. Berlin, Heidelberg, New York: Springer 1981

4. Botvich, D.D.: Proceedings of IY. Sov.-Jap. Symp. on Probability, 1982, In: Lecture Notes in Mathematics. Berlin, Heidelberg, New York: Springer 1983

5. Shale, D., Stinespring, W.F.: Ann. Math. 80, 365 (1964)

6. Maassen, H.: On the invertibility of Møller morphisms. J. Math. Phys. 23, 1848-1851 (1982)

7. Malyshev, V.A.: Usp. Math. Nauk 35, 3-51 (1980) (in Russian)

Communicated by Ya. G. Sinai

Received February 15, 1983 